\title{
Glutaric aciduria type 1: anaesthetic management of an adult patient for elective laparoscopic cholecystectomy
}

\section{Martins P. ${ }^{1}$, Oliveira L.. ${ }^{2}$, Ormonde L. ${ }^{3}$}

1 Trainee Anaesthetist; ${ }^{2}$ Staff Anaesthetist; ${ }^{3} \mathrm{Head}$ of Anaesthetic Department - Centro Hospitalar Lisboa Norte - Hospital de Santa Maria, Dept of Anaesthesiology \& Pain Medicine, Lisbon, Portugal

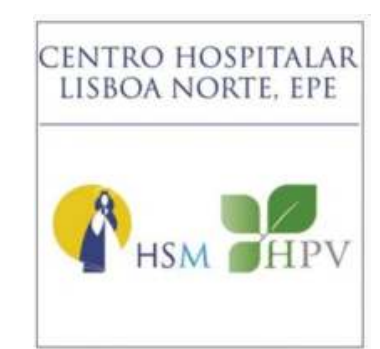

\section{Background}

Glutaric aciduria type 1 (GA1) is a rare hereditary metabolic disorder (prevalence of 1 in 100000 newborns).

Untreated, approximately $90 \%$ of patients will develop neurological disease following an acute encephalopathic crisis often precipitated by gastroenteritis, intercurrent febrile illness, immunization or surgical intervention.

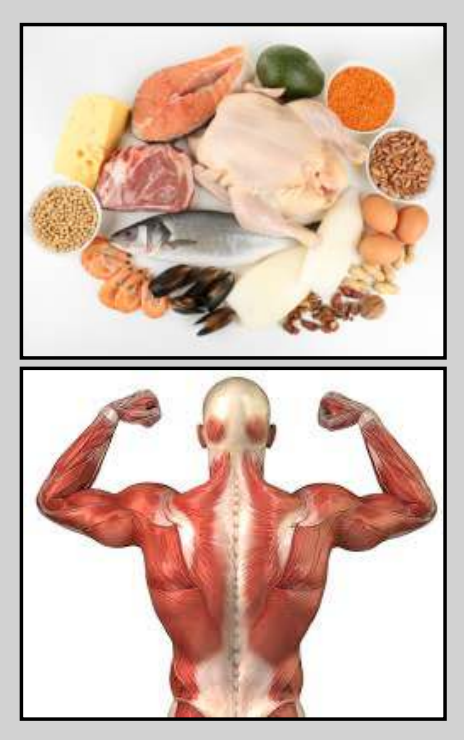

Health problems

\section{Case Report}

- $\sigma^{7}$, 20-year-old (weight $45 \mathrm{Kg}$, height $165 \mathrm{~cm}$ ) submitted to elective laparoscopic cholecystectomy

- Personal history: GA1 diagnosed at 1-year-old treated with low protein diet, riboflavin and carnitine. No acute metabolic disturbances in the past year

- Clinical examination: psychomotor delay, dystonia and dysarthria. Normal routine laboratory tests

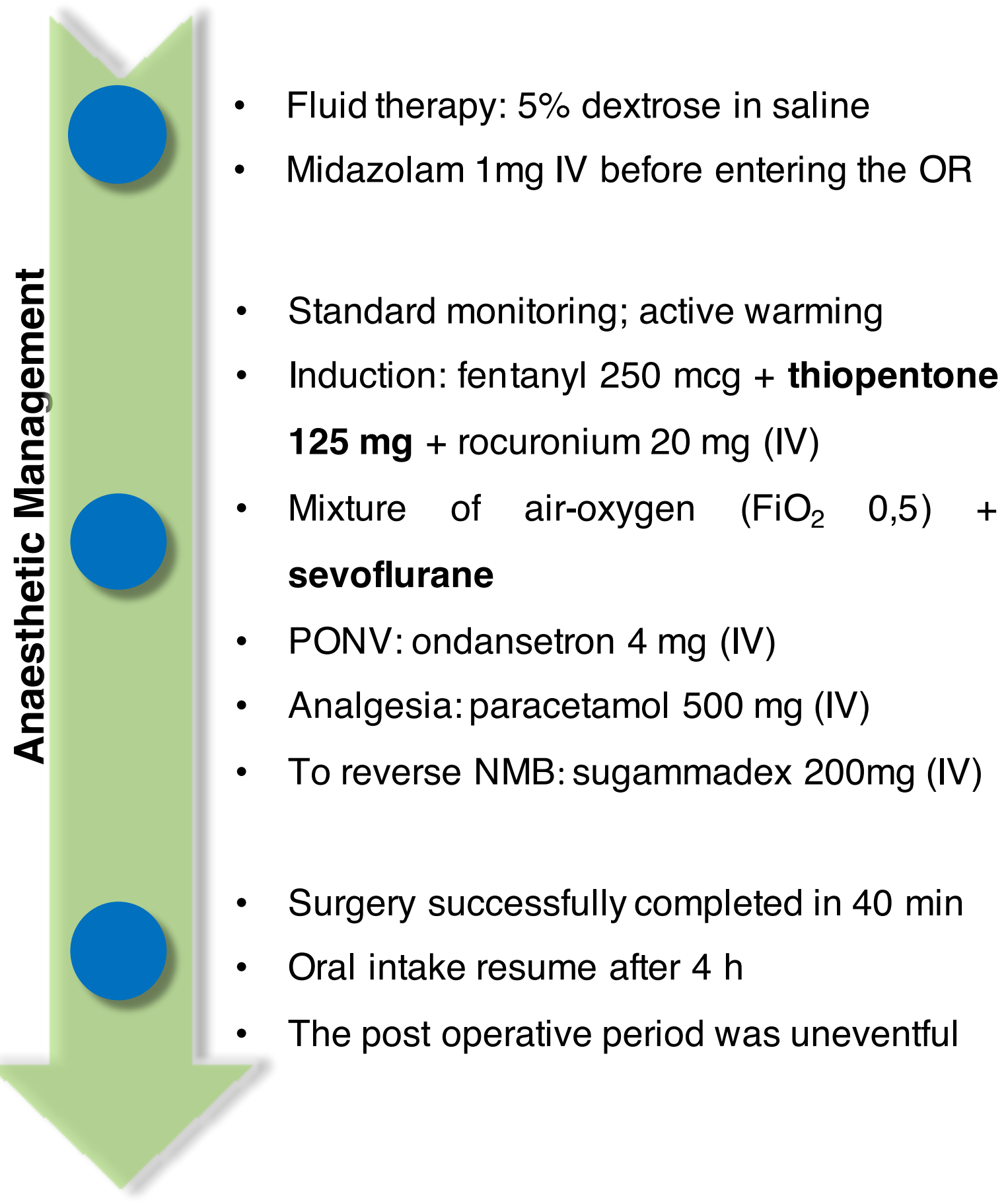

\section{Discussion}

Antecipate anaesthetic risks in GA1 patients:

- Dehydration

- Hyperthermia and catabolic state

- Possibility of pulmonary aspiration

- Prolonged responses to nondepolarizing muscle relaxants

- Hyperkalemic responses to succinylcholine

Main question: volatile anaesthetic agents - yes or not?

Against the use of volatile anesthetics $\rightarrow$ possible association between mitochondrial dysfunction and malignant hyperthermia? More recent literature rules out episodes of malignant hyperthermia and other intraoperative events attributable to the general anesthesia with sevoflurane in paediatric patients with mitochondrial disease.

Against propofol $\rightarrow$ lipid overload and inhibition oxidative phosphorylation, carnitine palmitoyltransferase transport of longchain fatty acids, and b-oxidation of fatty acid in mitochondria.

Although the described case was successful, the anaesthetic management of patients with metabolic disorders like GA1 remains challenging and more clinical data are necessary to improve these patients outcomes.

\section{References:}

K. Stefan et al; Diagnosis and management of glutaric aciduria type I - revised recommendations; $\mathrm{J}$ Inherit Metab Dis (2011) 34:677-694; H.P. Joaquin et al; Anesthetic management in two siblings with glutaric aciduria type 1; Pediatric Anesthesia (2006) 16: 188-191; W.N. Teng et al; Anesthetic management of comprehensive dental restoration in a child with glutaric aciduria type 1 using volatile sevoflurane; Journal of the Chinese Medical Association 77 (2014) 548-551. 